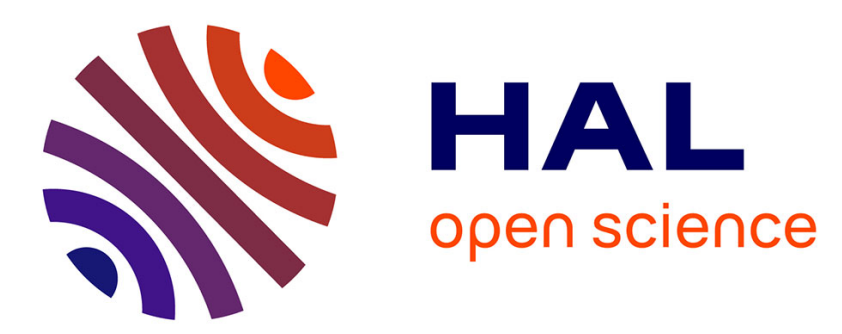

\title{
Simulating variable pitch crossflow water turbines: A coupled unsteady ONERA-EDLIN model and streamtube model
}

\author{
B. Paillard, F. Hauville, Jacques Andre Astolfi
}

\section{- To cite this version:}

B. Paillard, F. Hauville, Jacques Andre Astolfi. Simulating variable pitch crossflow water turbines: A coupled unsteady ONERA-EDLIN model and streamtube model. Renewable Energy, 2013, 52, pp.209-217. 10.1016/j.renene.2012.10.018 . hal-01081221

\section{HAL Id: hal-01081221 \\ https://hal.science/hal-01081221}

Submitted on 7 Nov 2014

HAL is a multi-disciplinary open access archive for the deposit and dissemination of scientific research documents, whether they are published or not. The documents may come from teaching and research institutions in France or abroad, or from public or private research centers.
L'archive ouverte pluridisciplinaire HAL, est destinée au dépôt et à la diffusion de documents scientifiques de niveau recherche, publiés ou non, émanant des établissements d'enseignement et de recherche français ou étrangers, des laboratoires publics ou privés. 


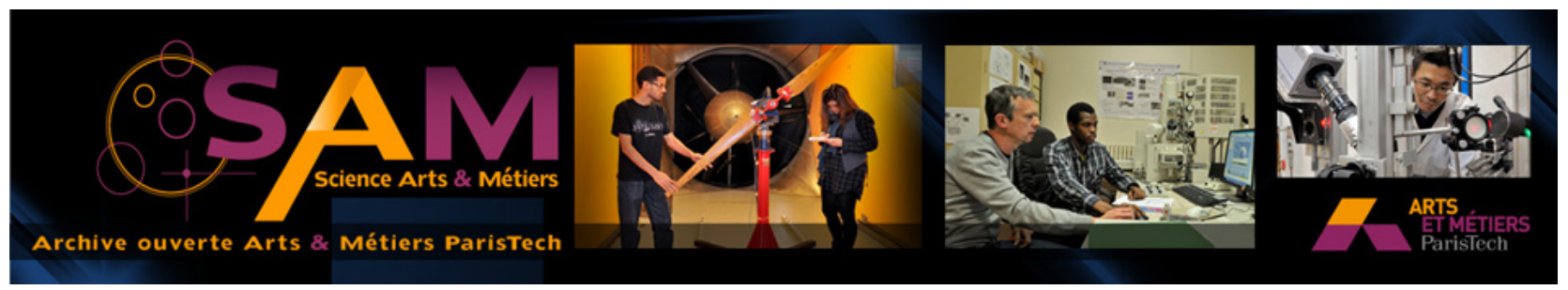

Science Arts \& Métiers (SAM)

is an open access repository that collects the work of Arts et Métiers ParisTech researchers and makes it freely available over the web where possible.

This is an author-deposited version published in: http://sam.ensam.eu

Handle ID: .http://hdl.handle.net/10985/8837

\section{To cite this version :}

B. PAILLARD, F. HAUVILLE, Jacques Andre ASTOLFI - Simulating variable pitch crossflow water turbines: A coupled unsteady ONERA-EDLIN model and streamtube model - Renewable Energy Vol. 52, p.209-217 - 2013 


\title{
Simulating variable pitch crossflow water turbines: A coupled unsteady ONERA-EDLIN model and streamtube model
}

\author{
B. Paillard*, F. Hauville, J.A. Astolfi

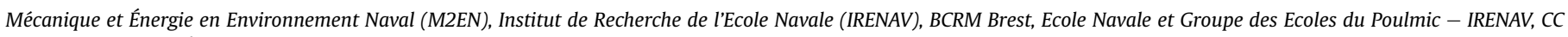 \\ 600, 29240 Brest Cedex 9, France
}

Keywords:

Darrieus

Variable pitch

Dynamic stall

Momentum theory

\begin{abstract}
A B S T R A C T
This article describes a new method for simulating unsteady hydrodynamics forces and moments on the blades of a crossflow 'Darrieus' turbine with active pitch variation. This method is based on the ONERAEDLIN dynamic stall model, coupled with a momentum streamtube model to take into account the turbine interference on the flow. Both models are presented, and compared separately with experimental results for a pitching airfoil for the ONERA-EDLIN model; and for Darrieus turbine for the momentum theory. The model coupling is then detailed and compared with experimental data taken from the open literature [1] The turbine has 2 straight blades with a NACA 0012 section operating in water at a mean chord Reynolds number of $4 \times 10^{4}$ for tip speed ratio $\lambda=2.5,5$ and 7.5. Good agreement was found for average $\lambda=5$, and qualitative agreement could be obtained at low and high $\lambda$, where dynamic stall effects and interference effects respectively are predominant. This is positive because $\lambda=5$ is the closest value from the optimal power production point. Variable pitch is finally introduced in the model and several functions are tested in order to increase efficiency. A maximum increase of $53 \%$ on the power coefficient was found to occur with a sinusoidal law.
\end{abstract}

\section{Introduction}

Tidal turbines are currently the power source that shows the most advantages [2]. No land occupation like a dam, steady predictable power input and output unlike wind turbines, no waste or side effects like fossil or nuclear power plants. These devices can consist of a classic horizontal axis screw-like systems, or crossflow turbines which have many advantages in water [3], such as being independent of the tide direction. Variable pitch crossflow turbines enable a Darrieus system to improve its performance and decrease parasitic forces, mainly responsible for fatigue and system failure [4]. They have been studied at IRENAV since 2007 as the SHIVA project. This project of novel tidal turbines deals with three topics, which will be introduced here. Darrieus turbines have been studied extensively during the 70s and 80s, especially by SANDIA organization [5-8]. A reference publication on this topic can be found in [9]. Though almost no Darrieus turbine produced electrical power from wind since early 90 s, a renewed interest arose from the development of water turbines because most drawbacks which prevented this

\footnotetext{
* Corresponding author.

E-mail addresses: ben.paillard@gmail.com (B. Paillard), frederic.hauville@ecolenavale.fr (F. Hauville), jacques-andre.astolfi@ecole-navale.fr (J.A. Astolfi).
}

system from becoming a major wind turbine system do not exist in water, as for instance major difficulties in protecting the Darrieus turbine from extreme wind conditions that are not encountered in tidal flows. Moreover the centrifugal forces are not as severe as they are in air [10]. For this reason many publications tackling various issues in water crossflow turbines were written in the past few years [11-13]. The first fluid model that was used for simulating the flow around a crossflow turbine range from simple momentum theory, where the turbine is divided in several streamtubes in which an actuator disk model is applied [14]. This model has been improved with the addition of many secondary effects. However it could never accurately simulate local forces on blades, only global performance through efficiency curves which was used as a first approach for design. The second method is a vortex theory [7] which could reach better local simulation, but remained limited as well because accurate stalled situation cannot be modeled, dynamic or static. Finally RANS, LES and DES methods were tried and gave better local results [15], but at the cost of a much longer solving time. Compared with the two first methods, for which CPU time is of the magnitude of a minute, these simulations give a complete solution for a CPU time ranging from a week to several months.

Dynamic and static stall characteristics of an airfoil have a very strong influence on the turbine performance. Considering how the 


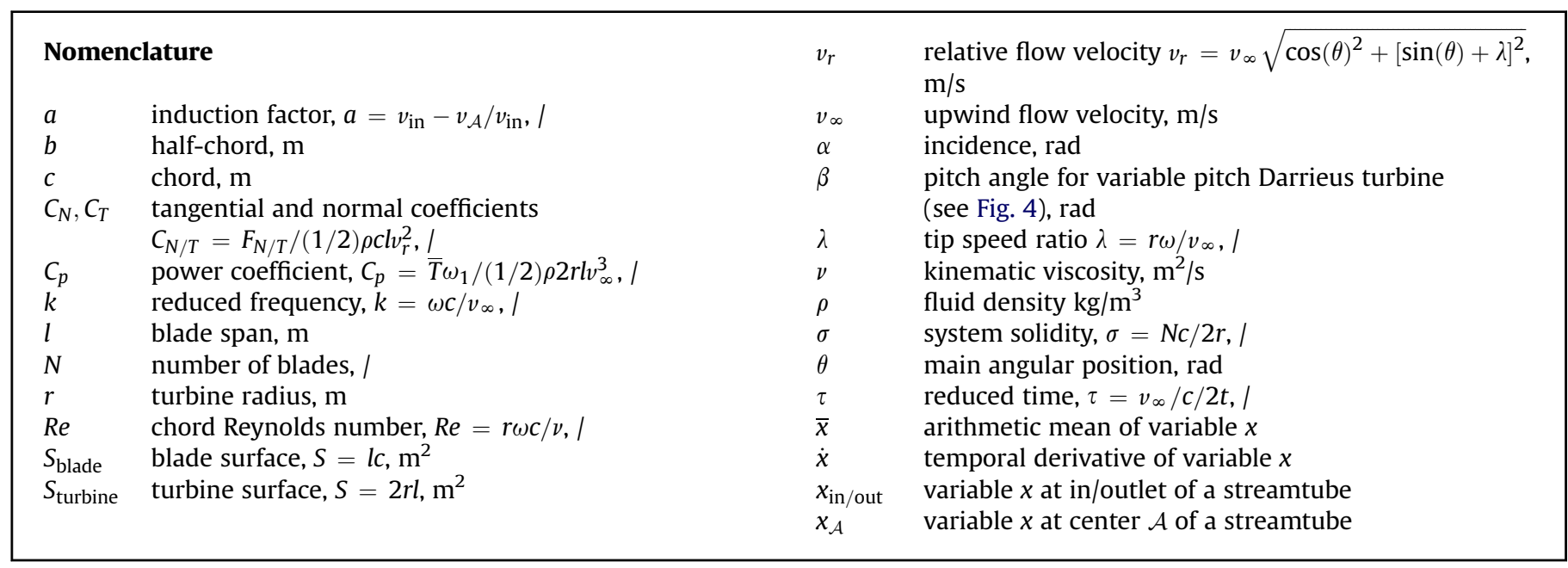

vortex method could not predict it accurately, and the complexity of a CFD simulation in an optimization process, the ONERA-EDLIN model is a very interesting compromise. On top of that, it has the ability to model any special kinematics and not just only pitch; it can predict unstalled dynamic behavior based on a potential formulation; and it can calculate aerodynamic moment when dynamic stall exist, which is interesting in the case of variable pitch.

Variable pitch crossflow turbines have mainly been developed as propellers. Their ability to create a thrust force in any direction enables a better control of ships. Nowadays the Voith-Schneider system [16] is often used when maneuverability is needed. All these devices can be classified according to their tip speed ratio $\lambda$. For wind/water turbines application, the $\lambda<1$ devices were tested and showed poor performances [17], thus only $\lambda>1$ devices are studied in this paper.

This paper focuses on the study of active control devices, for which each azimuthal position gives one pitch position. Passive devices on the other hand use springs and masses, taking advantage of centrifugal forces throughout turbine acceleration [13,18]. Recent research on active pitch control devices can be found. Ref. [19] used a modified CAARDAV code, the best double multiple streamtube model currently available. They simulated three kinds of pitch laws. The first one was the ideal one, which kept the incidence at a constant ideal value throughout the revolution, which resulted in a very steep pitch function. The second one just limited the angle of incidence to this ideal value to avoid any stall, resulting in a smoother curve. Finally the third law was a sine function which made the angle of attack continuously change from 0 to this ideal value, resulting in the smoothest curve. The performance was improved, and the final conclusion was that though a sine function delivers less power, it is much more desirable as far as fatigue and parasitic forces are concerned. An optimal sine amplitude had yet to be found since with the complex Darrieus kinematics the geometric incidence is not an explicit value, and it is thus difficult to choose an ideal angle of attack.

A Korean project [20] performed RANS simulation on a device with individual pitch control obtained by a servo motor on each blade. They obtained an ideal pitch function and a sinusoidal approximation of it. The system is currently under experimental test. In Scotland a project involving hydraulic variable pitch of the blades is being carried out [21]. Its main originality is to have a common pressure tank that would refill when the blade's hydrodynamic moment is oriented the same way as the blade rotational velocity, so that the actual energy cost of the pitch variation would be almost zero. A British project [22] is also under development, featuring hydraulic pitch control in a sine manner. It is however focused more on self-start than actual power improvement.

\section{Theoretical backgrounds}

The model studied here relies on two theories: the actuator disk, and the ONERA-EDLIN dynamic stall. Their basic concept will be introduced here.

\subsection{ONERA-EDLIN model}

For complete reference please refer to [23], as this is only a general introduction. This model is based on the idea that non linear systems, such as the aerodynamic coefficients, can be linearized for small amplitudes. Extending this idea to large amplitudes of variation around a steady value of the coefficient $C_{q, s}$ yields the Equation (1).

$a(\alpha) C_{q}+b(\alpha) \frac{\mathrm{d} C_{q}}{\mathrm{~d} t}+c(\alpha) \frac{\mathrm{d}^{2} C_{q}}{\mathrm{~d} t^{2}}=a(\alpha) C_{q, s}+e(\alpha) \frac{\mathrm{d} \alpha}{\mathrm{d} t}+f(\alpha) \frac{\mathrm{d}^{2} \alpha}{\mathrm{d} t^{2}}$

where $a, b, c, d, e$ and $f$ are explicit functions of $\alpha ; C_{q}$ is any of the three aerodynamic coefficients and $C_{q, s}$ its steady state value.

The strong differences between attached and detached flow required the use of two separate equations defined in Equations (3) and (4). The global force or moment is eventually obtained through summation of attached and detached components as defined in Equation (2).

global force or moment $Q=Q_{1}+Q_{2}$

attached flow $\left\{\begin{array}{ccc}\frac{\mathrm{d} Q_{1}}{\mathrm{~d} t}+\lambda Q_{1} & =\lambda Q_{\text {linear }}+(\lambda s+\sigma) \frac{\mathrm{d} \alpha}{\mathrm{d} t}+s \frac{\mathrm{d}^{2} \alpha}{\mathrm{d} t^{2}} & \text { lift } \\ Q_{1} & =Q_{\text {linear }}+(s+\sigma) \frac{\mathrm{d} \alpha}{\mathrm{d} t}+s \frac{\mathrm{d}^{2} \alpha}{\mathrm{d} t^{2}}\end{array} \quad\right.$ drag and moment 
detached flow $\frac{\mathrm{d}^{2} Q_{2}}{\mathrm{~d} t^{2}}+a \frac{\mathrm{d} Q_{2}}{\mathrm{~d} t}+r Q_{2}=-\left[r \Delta Q+E \frac{\mathrm{d} \alpha}{\mathrm{d} t}\right]$

The variables $\lambda, s, \sigma, a, r$ and $E$ are extracted from the thin foil theory or from experimental data. The term $Q_{2}$ which manages the stalled behavior is 0 before stall, and then increase at stall. This is controlled with the right hand term of Equation (4) which is 0 before stall. $\Delta Q$ is the difference between the extrapolated unstalled behavior into the stalled region (linear for lift, quadratic for drag, constant for moment). In order to account for a stall delay [24] this value is kept at zero for a constant reduced time $\Delta \tau_{d}$ after stall ( 8 for lift, 0 for drag and 2 for moment). This behavior is described in Fig. 1 for the lift coefficient.

After testing and modifying the base equations described above, Petot [23] introduced three formulations for lift, drag and moment unsteady prediction. They are described respectively in Equations (5)-(7) in the coefficient form.

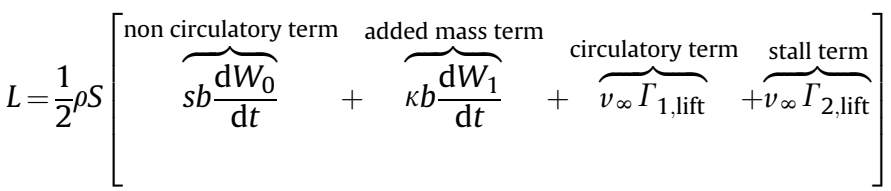

$D=\frac{1}{2} \rho S\left[v_{\infty}^{2} C_{d, \text { lin }}+\sigma b \frac{\mathrm{d} W_{0}}{\mathrm{~d} t}+v_{\infty} \Gamma_{2, \mathrm{drag}}\right]$

$M=\frac{1}{2} \rho S c\left[v_{\infty}^{2} C_{m, \text { lin }}+\bar{\sigma} b \frac{\mathrm{d} W_{0}}{\mathrm{~d} t}+\bar{\sigma} v_{\infty} W_{1}+s b \frac{\mathrm{d} W_{1}}{\mathrm{~d} t}\right.$

$$
\left.+v_{\infty} \Gamma_{2, \text { moment }}\right]
$$

$\Gamma_{1}$ and $\Gamma_{2}$ are obtained from differential Equations (8) and (9) respectively. $\Gamma_{1}$ is only required for lift calculation.

$$
\begin{aligned}
\frac{\mathrm{d} \Gamma_{1, \text { lift }}}{\mathrm{d} t}+\Lambda \frac{v_{\infty}}{b} \Gamma_{1, \text { lift }}= & \Lambda \frac{v_{\infty}}{b} p_{0} W_{0}+\Lambda \sigma \frac{v_{\infty}}{b} W_{1}+\left(\chi p_{0}+d\right) \frac{\mathrm{d} W_{0}}{\mathrm{~d} t} \\
& +\chi \sigma \frac{\mathrm{d} W_{1}}{\mathrm{~d} t}
\end{aligned}
$$

$$
\frac{\mathrm{d}^{2} \Gamma_{2, q}}{\mathrm{~d} t^{2}}+a \frac{v_{\infty}}{b} \frac{\mathrm{d} \Gamma_{2, q}}{\mathrm{~d} t}+r \frac{v_{\infty}^{2}}{b^{2}} \Gamma_{2, q}=-\left[r \frac{v_{\infty}^{2}}{b^{2}} V \Delta C_{q}+E \frac{v_{\infty}}{b} \frac{\mathrm{d} W_{0}}{\mathrm{~d} t}\right]
$$

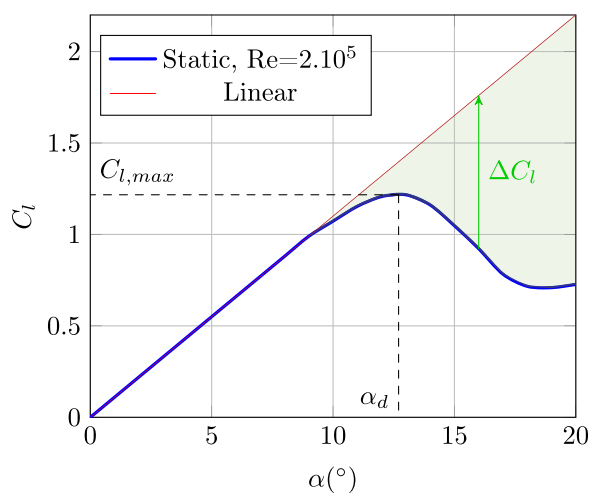

The variables $W_{0}$ and $W_{1}$ are of prime concern since they describe the kinematics of the foil. They are respectively the induced velocity at quarter chord, and the derivative of this induced velocity relatively to the half-chord variable. This is written in Equations (10) and (11).

$W_{0}=\frac{\partial \Phi}{\partial y}\left(x_{M} / c=0.25\right)$

$W_{1}=\frac{\partial \frac{\partial \Phi}{\partial y}}{\partial\left(x_{M} / b\right)}$

The zero normal velocity condition at wall enables the calculation of these variables as a function of the kinematics encountered. In our cases they are written in Equations (12) and (13).

pitching airfoil $\left\{\begin{array}{l}W_{0}=v_{\infty} \alpha \\ W_{1}=\frac{c}{2} \alpha\end{array}\right.$

Darrieus with variable pitch $\left\{\begin{array}{l}W_{0}=-v_{\infty} \cos (\theta+\beta)+r \dot{\theta} \sin (\beta) \\ W_{1}=\frac{c}{2}(\dot{\theta}+\dot{\beta})\end{array}\right.$

The differential systems are solved with the software Scilab using a 4th order Runge Kutta scheme.

Experimental data were used to validate this model [25] for lightly stalled and deeply stalled state. The experimental variables are gathered in Table 1. Agreement is good with max deviation below $50 \%$ at peaks during stall and below 10\% elsewhere. Results are shown in Fig. 2.

\subsection{Streamtube model}

This model is derived from the momentum "actuator disk" model. It equates the lift and drag projection on the undisturbed flow axis with the fluid momentum decrease when going through the turbine. The main output of this model is the interference parameter defined by Equations (14) and (15), where $v_{\square}$ is the velocity of the fluid in the tube of fluid considered, $v_{\text {in }}$ is the velocity of the fluid upwind from this tube, and $v_{\text {out }}$ is the velocity of the fluid downwind from this tube

$v_{\mathcal{A}}=(1-a) v_{\text {in }}$

$v_{\text {out }}=(1-2 a) v_{\text {in }}$

Fig. 1. $\Delta C_{l}$ variable evolution as a function of $\alpha$ including stall delay. 
Table 1

Experimental data used for ONERA-EDLIN model validation using a pitching airfoil.

\begin{tabular}{|c|c|c|c|c|c|c|c|}
\hline Frame & 7108 & 7110 & 7111 & 9210 & 9213 & 9214 & 9217 \\
\hline Fluid & & & & Air at $20^{\circ}$ & & & \\
\hline Reynolds number & & $3.90 \times 10^{6}$ & & $\mid$ & $3.5 \times 10^{6}$ & & \\
\hline Kinematics & & & & Sinusoidal & & & \\
\hline Mean angle & & $8^{\circ}$ & & & $15^{\circ}$ & & \\
\hline Amplitude & & $10^{\circ}$ & & | & $20^{\circ}$ & & \\
\hline Frequency & $1.35 \mathrm{~Hz}$ & $5.40 \mathrm{~Hz}$ & $10.80 \mathrm{~Hz}$ & $0.53 \mathrm{~Hz}$ & $1.31 \mathrm{~Hz}$ & $2.62 \mathrm{~Hz}$ & $5.24 \mathrm{~Hz}$ \\
\hline Reduced frequency & 0.0250 & 0.0997 & 0.1992 & 0.0098 & 0.0243 & 0.0489 & 0.0983 \\
\hline Foil section & & & & NACA0012 & & & \\
\hline Chord & & & & $0.61 \mathrm{~m}$ & & & \\
\hline Rotation axis & & & & Quarter chord & & & \\
\hline
\end{tabular}

The turbine is divided in several tubes defined by the azimuthal step chosen. The turbine is also divided into an upstream and an downstream part, and $v_{\text {out }}$ from upstream computation becomes $v_{\text {in }}$ for downstream section. A typical tubes number for the cases considered is 20. The final formulation for computing the interference parameter $a$ is written in Equation (16), where $N$ is the number of blades, $C$ is the chord, $v_{r}$ is the relative fluid velocity in the foil frame of reference, $r$ is the turbine's radius, $\theta$ is the azimuthal position, $\beta$ is the blade pitch, $C_{N}$ and $C_{T}$ are the hydrodynamic coefficients, normal and tangential respectively.
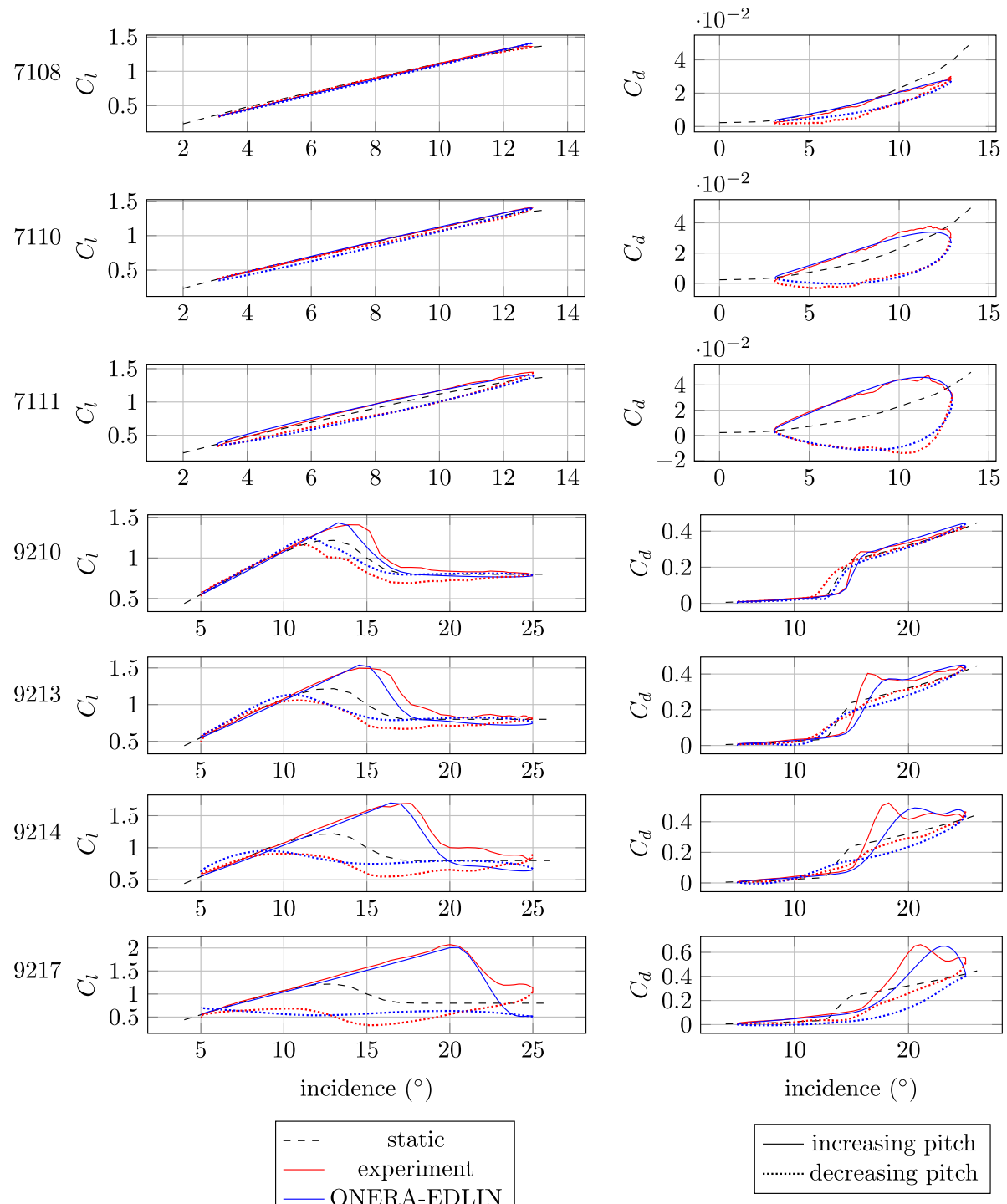

Fig. 2. Validation of ONERA-EDLIN model for various amplitude and frequency on a pitching airfoil. 
$a(1-a)=\frac{N c v_{r}^{2}}{8 \pi r|\cos (\theta)| v_{\mathrm{in}}^{2}}\left(C_{N} \cos (\theta+\beta)-C_{T} \sin (\theta+\beta)\right)$

The hydrodynamic coefficients need to be interpolated from data and modified to account for the dynamic effects due to blade motion. That is what is done with the ONERA-EDLIN model.

Solving Equation (16) is done with an iterative process, in our case a Newton's method, with an accuracy of $10^{-3}$. Convergence is usually obtained in less than 10 loops. This model has been improved through the years to account for several secondary effects such as tower interference or fluid expansion [26], resulting in the CARDAAV code. In our study though most of these effects were not taken into account because they did not provide much improvement, and involved a heavier calculation.

The main limitation of this model is the approximation of a laminar, steady flow. In the cases of heavy loadings or large solidities experiments show recirculation, vortices generation and other energy losses. In these cases the streamtube theories often cannot converge, or exhibit unphysical behavior such as diverging loads or interference factor. One must hence be very careful while using this model to avoid unphysical solutions.

Coupling the models requires considering several strategies. Whereas using a full Double Multiple Streamtube model is supposed to be more precise, it requires much more CPU power, because at every iteration loop in the evaluation of the interference parameter $a$ an ONERA-EDLIN simulation must be ran. Another option is the use of a Double Single Streamtube model, which divides the turbine in just two areas, one upstream and one downstream. Both models were compared as far as CPU performance, convergence and experimental agreement are concerned. Fig. 3 shows that the difference is minimal, for a CPU time multiplied by approximately 10 . Thus it was decided to use a double single streamtube rather that a double multiple.

\section{Comparison with experiment}

\subsection{Experimental data}

The experimental data are taken from [1]. Even though they were made at a relatively low Reynolds number they are relevant since they cover a wide range of tip speed ratios including an operational one. No experimental data could be found at a higher Reynolds number at the time the article was written. It will be
Table 2

Experimental cases taken from [1] used in this article.

\begin{tabular}{llll}
\hline & Case 1 & Case 2 & Case 3 \\
\hline Fluid & & Water & \\
Undisturbed fluid velocity & $0.183 \mathrm{~m} / \mathrm{s}$ & $0.091 \mathrm{~m} / \mathrm{s}$ & $0.061 \mathrm{~m} / \mathrm{s}$ \\
Number of blades & & 2 & \\
Diameter & & $1.22 \mathrm{~m}$ & \\
Span & $1.1 \mathrm{~m}$ & \\
Chord & $0.0914 \mathrm{~m}$ & 7.5 \\
Total solidity & 0.3 & \\
Blade section & NACA0012 \\
Rotational speed & $43 \mathrm{rpm}=0.75 \mathrm{rad} / \mathrm{s}$ & \\
Tip speed ratio $\lambda$ & & 5 & \\
Chord Reynolds number & 2.5 & $4 \times 10^{4}$ & \\
\hline
\end{tabular}

needed however since full scale turbines will run at a higher Reynolds number. The data are gathered in Table 2 .

The measured values in this study are the normal and tangential force coefficients defined by Equations (17) and (18). They are relevant in the study of crossflow turbines since they are respectively parasitic and useful forces. The coefficient of performance is proportional to the coefficient of tangential force as described by Equation (19) [27]. This makes $C_{T}$ a relevant parameter for performance evaluation. In the present study performance increase will be directly associated with $C_{T}$ increase.

$$
\begin{aligned}
& C_{N}=\frac{F_{N}}{\frac{1}{2} \rho S_{\text {blade }} V_{\infty}^{2}} \\
& C_{T}=\frac{F_{T}}{\frac{1}{2} \rho S_{\text {blade }} V_{\infty}^{2}}
\end{aligned}
$$

$C_{p}=\sigma \lambda \overline{C_{T}}$

The coordinate system used in this study is illustrated in Fig. 4.

\subsection{Results and discussion}

Comparison of the results given by the ONERA-EDLIN simulation and experiment is shown in Fig. 5. On this figure is also displayed a classical DMST with Gormont dynamic stall from [17]. The agreement exhibits better general prediction for tangential coefficient $C_{T}$, especially for the upstream section. For case $2(\lambda=5)$ the

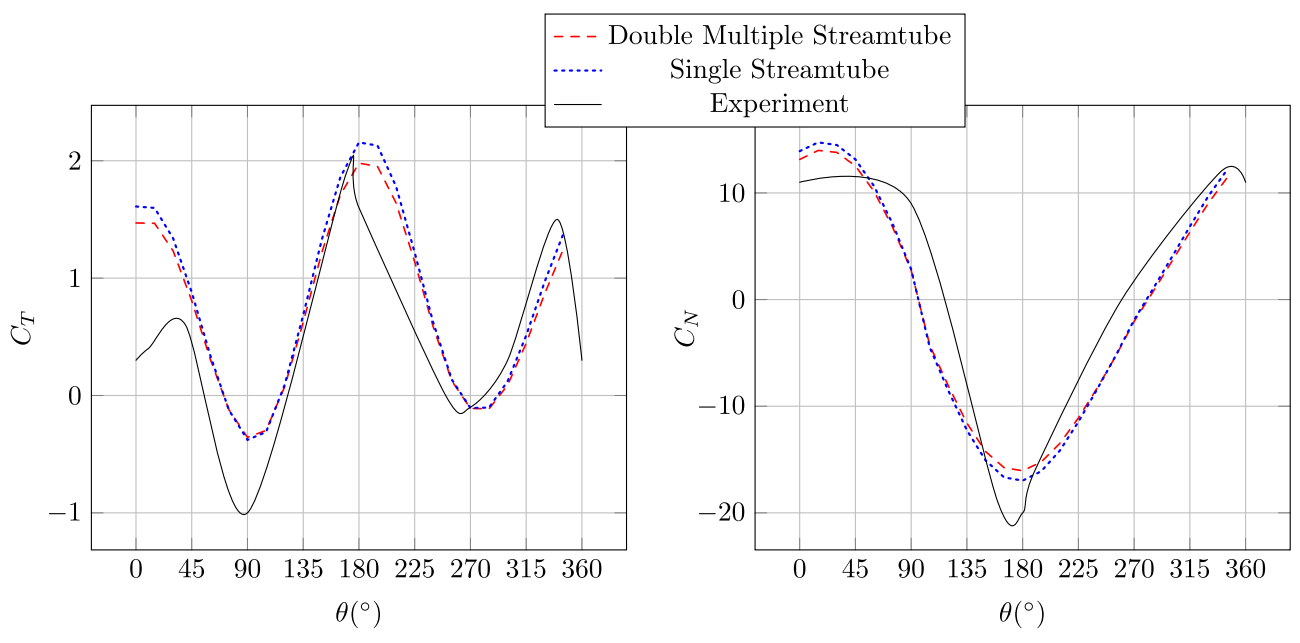

Fig. 3. Single streamtube versus double streamtube method for case $2(\lambda=5)$. 

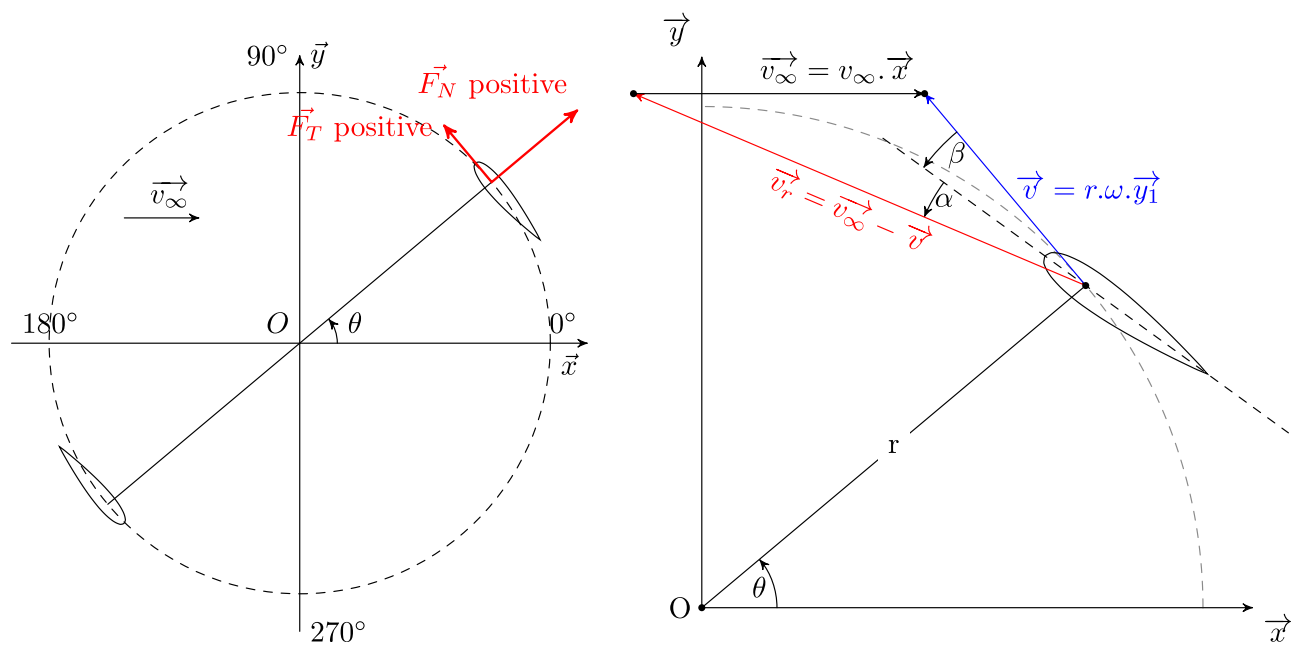

Fig. 4. Coordinate system used in this study.

prediction is good, general behavior is correctly simulated and most extremums are predicted well. The only issue is the downstream part for which the model predicts a high torque whereas experimental data show a steep decrease around $340^{\circ}$. This is due to the energetic model and shows that it might be unable to predict a correct interference. For case $1(\lambda=2.5)$ and $3(\lambda=7.5)$ agreement is not qualitatively as good. The same over-prediction occurs at the downstream part, and normal forces are under-predicted. The worst case is case 1 for which $\lambda$ is the lowest. This was predictable since it is the case where the incidence is highest, where the stall is deepest. Even though the tangential force is predicted better with the ONERA-EDLIN that with DMST, a difference still exists, which would require improving the stall model further (Fig. 5).

Case $2(\lambda=5)$ being the case which is the closest from an operational point, its agreement with experimental results is positive regarding the use of ONERA-EDLIN model coupled with
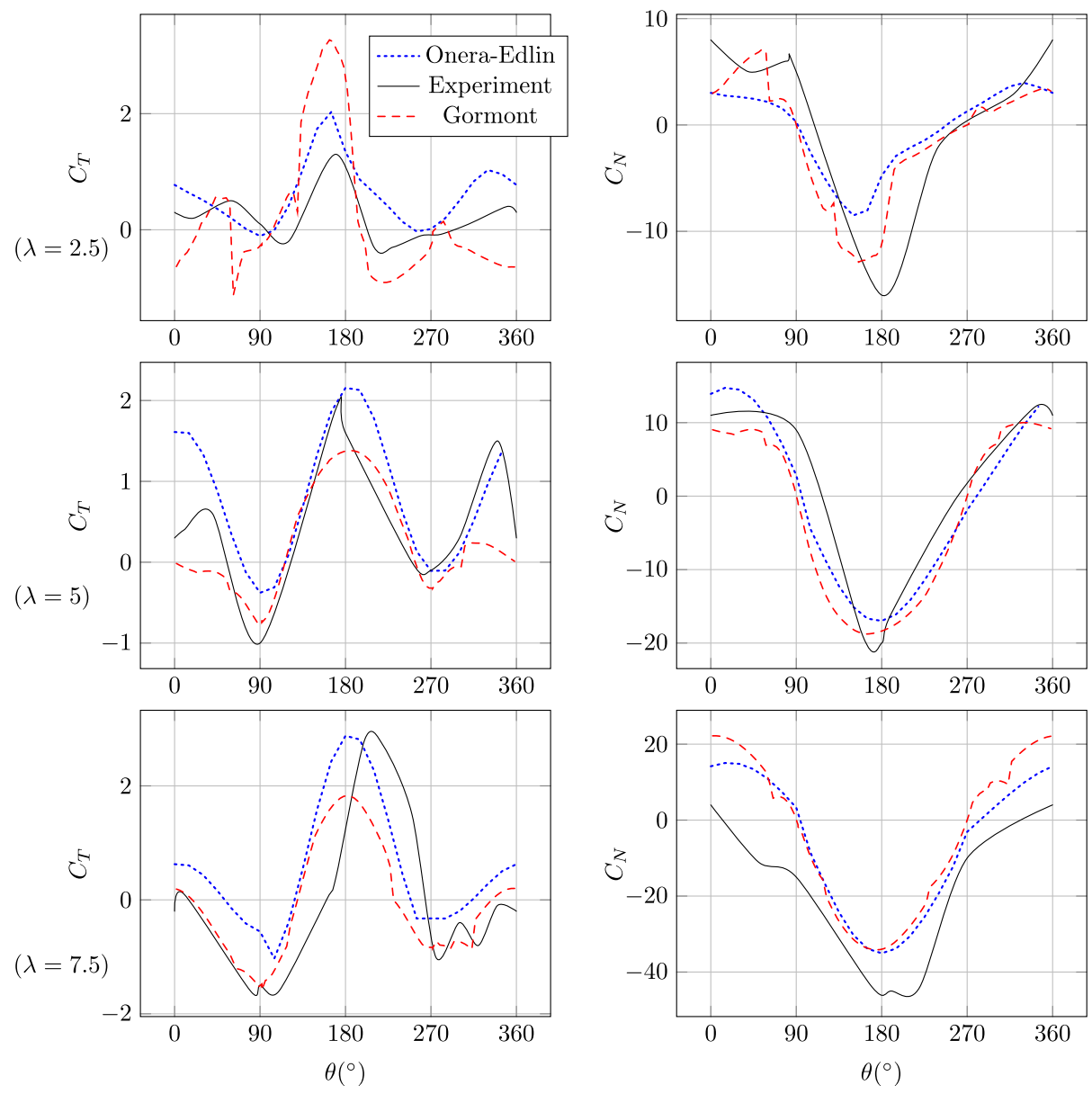

Fig. 5. Comparison of ONERA-EDLIN model with experiment and DMST-Gormont. 


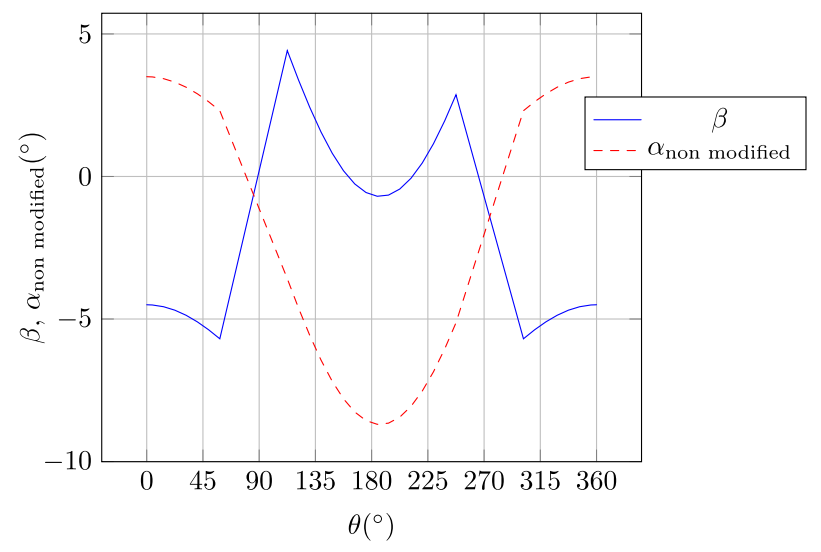

Fig. 6. Pitch and incidence angles considered in constant pitch function predicted by DMST method.

single streamtube model. We will now use this case as a test case for variable pitch.

\section{Variable pitch}

Two strategies were used in this study to find an appropriate pitch law. First the constant incidence law as described by [19] was tried. It aims at maintaining the local incidence at a constant angle slightly below the stall angle. The foil has to undergo a pressure/ suction side shift during upstream/downstream transitions. Considering a goal incidence $\alpha_{\text {goal }}$, the actual incidence would then be $-\alpha_{\text {goal }}$ on the upstream part and $+\alpha_{\text {goal }}$ on the downstream part (or vice versa). This is going to require a very high angular acceleration though, which might not be desirable. This is why a sinusoidal law is then tried. $\alpha_{\text {goal }}$ is used as maximum and minimum values, with a sinusoidal variation between them. Such a pitch variation will be more easily implemented, and will be preferable if performance increase remains high enough compared with the constant incidence law.

It is important to note that no experimental data could be found for variable pitch devices, which means that the ONERA-EDLIN model could not be validated in this case. However the kinematic of a crossflow Darrieus turbine simulated by the present model has been validated, and the only difference for variable pitch modelization is a small change in pitch. Several tip speed ratio, hence several incidence functions, have been validated, and this was considered satisfactory to start exploring the behavior of variable pitch crossflow turbines. It is however clear that experimental data are required, which is why a variable pitch Darrieus experimental device is under construction at IRENAV.

\subsection{Constant incidence function}

Constant incidence is obtained with a $\beta$ function drawn as a function of the azimuthal position in Fig. 6. On this figure is also drawn the incidence found without any pitch variation, and the final incidence is then $\beta-\alpha$ non modified. The $\beta$ curve shown in Fig. 4 was found through a tangential force optimization reported in [17], which suggested that $\alpha_{\text {goal }}$ should be set to $8^{\circ}$. This value was found to be above optimal lift-to-drag ratio, but below stall angle. The same kind of optimized pitch was reported in [21] and [28]. On top of that a smoother linear variation was used at upstream/downstream transition to ensure convergence of the differential equations solver as a part of the ONERA-EDLIN model.

Fig. 7 shows the results obtained with the present model with fixed and constant pitch function, compared with Gormont model with constant incidence function as used by [28], and experimental results. A $33 \% C_{T}$ increase is simulated by ONERA-EDLIN model, which is much lower than DMST prediction. The latter predicts a steep change and a plateau in $C_{T}$ right after upstream/downstream part which looks similar to a static model; whereas ONERAEDLIN model predicts a smooth increase toward its maximal value. This seems much more physical considering the added mass effects involved, and the time needed for a steady state to develop fully around the foil.

In order to set the blades in pitch motion around their quarter chord, and since some hydrodynamic moment exist, some power source is required. The ONERA-EDLIN model is able to accurately predict unsteady hydrodynamic moment of a blade $M_{\text {hydro, }}$ a variable known to be highly influenced by dynamic stall. When multiplying this moment with the rotational velocity $\dot{\beta}$, one can calculate how much power goes into changing pitch. The pitch variation power is a dimensionalized so that it can be compared with the power coefficient of the turbine $C_{p}$. The non dimensional pitch motion power, namely $C p_{\text {pitch variation, is obtained through }}$ Equation (20). The hydrodynamic moment coefficient $C_{m \text {,hydro }}$ during revolution and $C p_{\text {pitch variation }}$ are shown in Fig. 8.

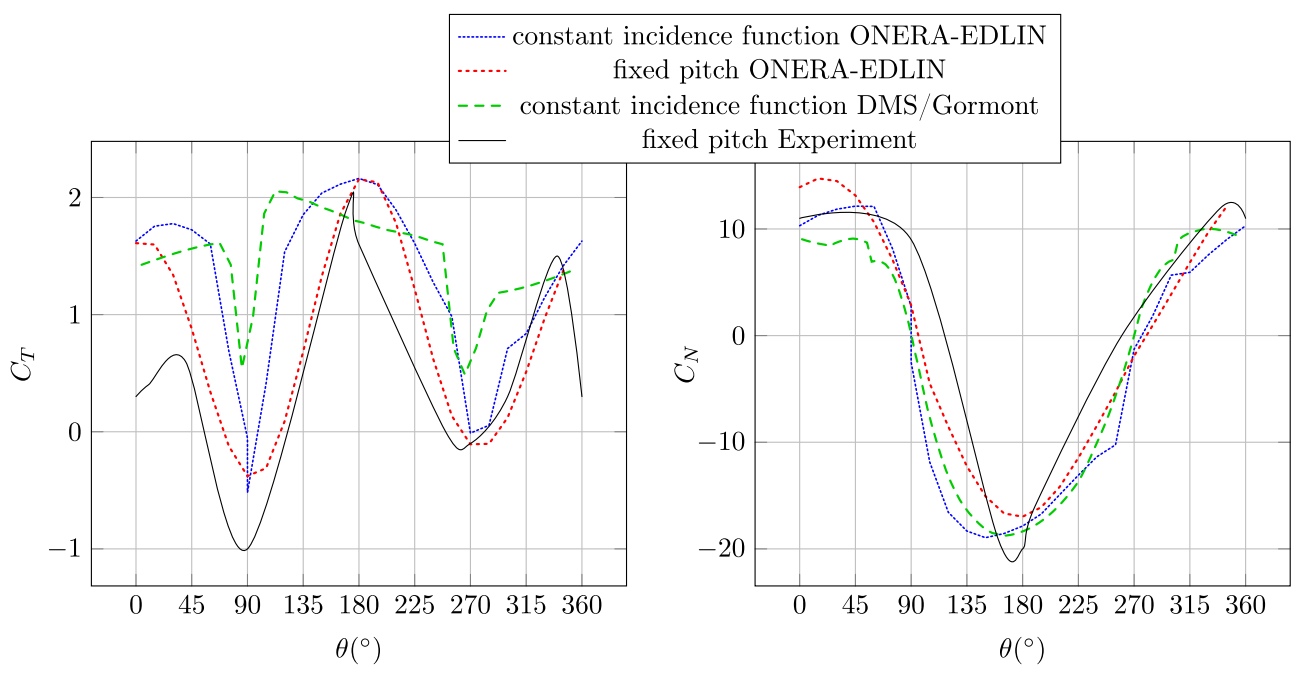

Fig. 7. Results of constant incidence function applied to case $2(\lambda=5)$. 

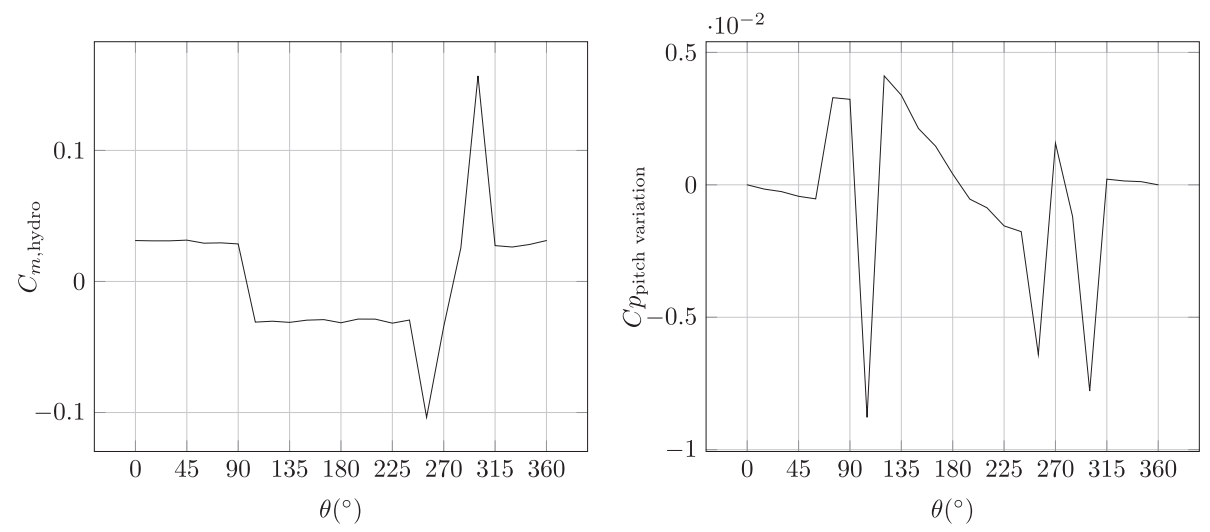

Fig. 8. Hydrodynamic moment calculated by ONERA-EDLIN model and its influence on coefficient of performance.
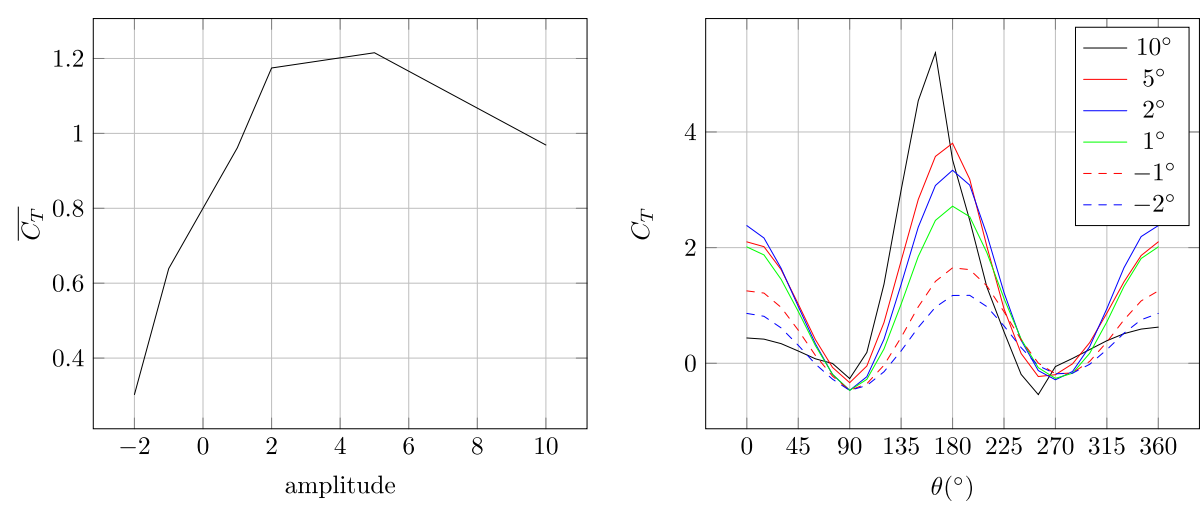

Fig. 9. Sine pitch functions simulated by ONERA-EDLIN model, case $2(\lambda=5)$.

$C p_{\text {pitch variation }}=\frac{M_{\text {hydro }} \dot{\beta}}{\frac{1}{2} \rho S_{\text {turbine }} v_{\infty}^{3}}$

$=\frac{c^{2} v_{r}^{2} C_{m} \dot{\beta}}{2 r v_{\infty}^{3}}$

In Fig. $8, C p_{\text {pitch variation is positive when power is required to set }}$ the blade in rotation around its quarter chord; oppositely $C p_{\text {pitch }}$ variation is negative if the moment is in the appropriate direction to change the foil pitch according to the pitch function chosen. We can see immediately that steps in pitch produce strong effects on the power associated with pitch change. For the moment coefficient a peak only appears during the transition upstream $\mapsto$ downstream part, which is explained by the fact that the stall model is triggered there, due to the lower local velocity. The $C p_{\text {pitch }}$ variation value remains low at all time however, always lower than $1 \%$, which is insignificant compared to a device for which the mean power coefficient is around 30\%. The mean value of $C p_{\text {pitch }}$ variation is $0.00767 \%$. With a system that can actually use power when this coefficient is negative, we realize that a variable pitch would have no effect on the performances. A mechanical system (gears, rods...) or a hydraulic system with pressure tank is one of these systems. On the other hand a hydraulic system without pressure tank, or a electrical servo motor device will not be able to take advantage of these negative values; in this case the mean absolute value needs to be considered: $0.23 \%$ here, which remains insignificant.
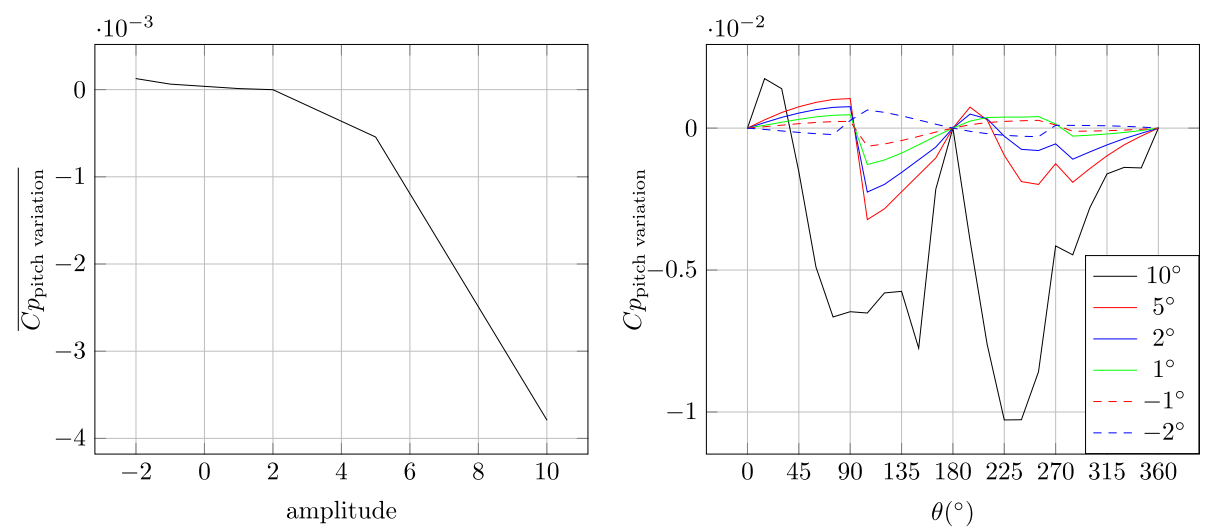

Fig. 10. Hydrodynamic moment of blades during sine pitch function simulated by ONERA-EDLIN model. 


\subsection{Sinusoidal pitch function}

As described in [19] the steep variation in pitch angle makes it difficult to implement. Hence we will use sine functions to try and come as close as possible to the performance increase obtained with a constant incidence function. This sine function will have the same frequency as the turbine frequency. It will be positive when the leading edge pitches inward in upstream section and outward in downstream section, resulting in incidence increase in both areas. A loop was used to run simulation for several amplitudes. Fig. 9 shows performances obtained with these sine pitch function, and Fig. 10 shows the influence on the moment coefficient. The performance is indeed increased with a sine pitch law. However the ideal function introduced by [19] and confirmed by [17] is in strong disagreement with what is obtained here. The ONERA-EDLIN model predicts a maximum power production point for an amplitude of $+5^{\circ}$, which would result in an incidence of $13^{\circ}$, far above the stall angle of $9^{\circ}$ at this Reynolds number. Compared with the fixed pitch device for which amplitude is 0 , the $+5^{\circ}$ amplitude gives a $\overline{C_{T}}$ increase of $51 \%$, raising from 0.8 to 1.21 . Dynamic stall is known to produce lift overshoots which in this case enable the system to reach a higher level of performance. The stalled state observed after dynamic stall phenomenon is not observed here due to the transition upstream $\mapsto$ downstream part. The pressure/suction side shift seems to dampen the dynamic stall negative consequences according to ONERA-EDLIN model. An amplitude of $10^{\circ}$ gives an even bigger peak, but at a cost of a largest decrease due to a deeper stall, and a disturbed downstream area which results in lower performance. On Fig. 10 the moment is shown. It is once more insignificant as far as performance is concerned, except for a $10^{\circ}$ amplitude for which it reaches $1 \%$ during peaks. The moment coefficient seems to be a decreasing function of the sine amplitude.

\section{Conclusion}

A coupling method between the ONERA-EDLIN model and a streamtube model has been implemented successfully to model the performance of a Darrieus-like turbine with active pitch variation. Good agreement is found with experimental results obtained from the open literature for force coefficients at the operational tip speed ratio of about 5 for a zero fixed pitch. It is not fully satisfactory for lower and higher tip speed ratios, even though an improvement compared with DMST/Gormont dynamic stall model was found. This deviation requires further investigation of the coupling method. Stall model improvement through model constants tends to improve agreement with experimental results at low tip speed ratio. At high tip speed ratio the energetic model is likely to be the origin of discrepancy. Concerning pitch variation, two scenarios were implemented. Sinusoidal functions exhibited a performance increase associated with dynamic stall phenomenon, for which maximum rise was found to be $+51 \%$. On the other hand power consumption required for blade pitch variation was shown to be non significant. This justifies further studies on variable pitch devices. A CFD model is currently being studied for further flow understanding, and an experimental device aiming at validating the different models with variable pitch is under construction.

\section{References}

[1] Strickland J, Webster B, Nguyen T. A vortex model of the Darrieus turbine: an analytical and experimental study. Journal of Fluids Engineering 1979;101: 500-5.

[2] Khan M, Bhuyan G, Iqbal M, Quaicoe J. Hydrokinetic energy conversion systems and assessment of horizontal and vertical axis turbines for river and tidal applications: a technology status review. Applied Energy 2009;86(10): $1823-35$.

[3] Kiho S, Shiono M, Suzuki K. The power generation from tidal currents by Darrieus turbine. Renewable Energy 1996;9(1-4):1242-5.

[4] Kirke B, Lazauskas L. Limitations of fixed pitch Darrieus hydrokinetic turbines and the challenge of variable pitch. Renewable Energy 2010;36:893-7.

[5] Blackwell B, Reis G. Blade shape for a tropskien type of vertical-axis wind turbine. Tech. Rep.; SLA-74-0154. Albuquerque, NM (USA): Sandia Labs; 1977.

[6] Sheldahl R, Klimas P. Aerodynamic characteristics of seven symmetrical airfoil sections through 180 degree angle of attack for use in aerodynamic analysis of vertical axis wind turbines. Tech. Rep. SANDIA Laboratories; 1981.

[7] Strickland J, Webster B, Nguyen T. Vortex model of the Darrieus turbine: an analytical and experimental study. Tech. Rep. SAND 79-7058. Albuquerque: Sandia National Laboratories; 1980.

[8] Wilson R, Walker S. Fixed-wake analysis of the Darrieus rotor. Tech. Rep.; SAND-81-7026. Albuquerque, NM (USA): Sandia National Labs; 1981. Oregon State Univ., Corvallis (USA). Dept. of Mechanical Engineering.

[9] Paraschivoiu I. Wind turbine design: with emphasis on Darrieus concept. Canada: Polytechnic International Press; 2002.

[10] Zanette J, Imbault D, Tourabi A. A design methodology for cross flow water turbines. Renewable Energy 2010;35(5):997-1009.

[11] Fraunie P, Beguier C, Paraschivoiu I, Brochier G. Water channel experiments of dynamic stall on Darrieus wind turbine blades. Journal of Propulsion and Power 1986;2:445-9.

[12] Klaptocz V, Rawlings G, Nabavi Y, Alidadi M, Li Y, Calisal S. Numerical and experimental investigation of a ducted vertical axis tidal current turbine. In: Proceedings of the 7th European wave and tidal energy conference; 2007. Porto, Portugal.

[13] Coiro D, De Marco A, Nicolosi F, Melone S, Montella F. Dynamic behaviour of the patented kobold tidal current turbine: numerical and experimental aspects. Acta Polytechnica 2005;45(3):77-84.

[14] Strickland JH. The Darrieus turbine: a performance prediction model using multiple streamtubes. Tech. Rep. Sandia Laboratories; 1975.

[15] Ferreira C, Bijl H, Bussel G, Kuik G. Simulating dynamic stall in a 2d vawt: modeling strategy, verification and validation with particle image velocimetry data. In: Journal of physics: conference series, vol. 75. IOP Publishing; 2007. p. 012023.

[16] Bartels J, Jürgens D. Latest developments in Voith Schneider propulsion systems. In: The 18th international tug \& salvage convention; 2004. Miami, FL.

[17] Paillard B, Hauville F, Astolfi J. Evaluation of active variable pitch technologies as current turbines. In: World renewable energy congress; 2008.

[18] Lazauskas L. Three pitch control systems for vertical axis wind turbines compared. Wind Engineering 1992;16(5):269-82.

[19] Staelens Y, Saeed F, Paraschivoiu I. A straight-bladed variable-pitch vawt concept for improved power generation. In: ASME 2003 wind energy symposium. 2003-524. ASME; 2003. p. 146-54.

[20] Hwang I, Lee Y, Kim S. Optimization of cycloidal water turbine and the performance improvement by individual blade control. Applied Energy 2009; 86(9):1532-40.

[21] Salter S, Taylor J. Vertical-axis tidal-current generators and the pentland firth. Proceedings of the Institution of Mechanical Engineers, Part A: Journal of Power and Energy 2007;221(2):181-99.

[22] Schönborn A, Chantzidakis M. Development of a hydraulic control mechanism for cyclic pitch marine current turbines. Renewable Energy 2007;32(4):662-79.

[23] Petot D. Modélisation du décrochage dynamique par équations différentielles. La Recherche Aerospatiale 1989;5:59-72.

[24] Beddoes T. A synthesis of unsteady aerodynamic effects including stall hysteresis. Vertica 1976;1(2):113-23.

[25] Mcalister KW, Carr LW, Mccroskey WJ. Dynamic stall experiments on the NACA 0012 airfoil. Tech. Rep. NASA; 1978.

[26] Paraschivoiu I, Delclaux F, Fraunié P, Beguier C. Aerodynamic analysis of the Darrieus rotor including secondary effects. Journal of Energy 1983;7(5):416-22.

[27] Paillard B. Simulation numérique et optimisation d'une hydrolienne à axe transverse avec contrôle actif de l'angle de calage. Ph.D. thesis; IRENAV; 2011.

[28] Paraschivoiu I, Trifu O, Saeed F. H-darrieus wind turbine with blade pitch control. International Journal of Rotating Machinery 2009;2009:1-7. 